Dans le monde...

\title{
Évolution récente du cycle du carbone planétaire : facteurs humains et naturels
}

L'accord de Paris, acté à la COP21, engage les États à réduire leurs émissions de gaz à effet de serre en général et de dioxyde de carbone $\left(\mathrm{CO}_{2}\right)$ en particulier d'ici 2025-2030. De tels engagements nous amènent à porter un regard attentif sur l'évolution récente du cycle du carbone global.

Le cycle du carbone naturel fait principalement intervenir l'atmosphère, les réservoirs de carbone organique de la végétation et des sols, les réservoirs de carbone inorganique, organique et le plancton de l'océan. Aux échanges naturels de $\mathrm{CO}_{2}$ entre ces réservoirs s'ajoutent d'importantes émissions de $\mathrm{CO}_{2}$ issues des activités humaines dues à l'utilisation d'énergies fossiles telles que le charbon, le gaz naturel et le pétrole, la fabrication de ciment et l'expansion de l'agriculture au détriment des écosystèmes naturels.

Une partie de ces émissions s'accumule dans l'atmosphère, tandis que le reste est absorbé dans les réservoirs naturels. Le temps d'absorption d'une émission instantanée de $\mathrm{CO}_{2}$ dans l'atmosphère est très long. Après 100 ans, entre 30 et $40 \%$ du $\mathrm{CO}_{2}$ initialement émis réside encore dans l'atmosphère selon la quantité émise ; après 1000 ans, il en reste $20 \%$ ou plus. Cela implique que l'augmentation de l'effet de serre lié aux émissions de $\mathrm{CO}_{2}$ actuelles et passées aura un impact sur le changement climatique futur sur de très longues échelles de temps.

Le Global Carbon Project ${ }^{1}$ estime que les émissions anthropiques de $\mathrm{CO}_{2}$ liées à l'utilisation d'énergies fossiles et à la fabrication de ciment ont été de 33 milliards de tonnes de $\mathrm{CO}_{2}$ par an en moyenne sur la période 2005-2014. Cette quantité est assez bien connue dans la mesure où elle est dérivée des statistiques énergétiques des pays. À cette quantité s'ajoutent les émissions de $\mathrm{CO}_{2}$ liées à l'utilisation des sols et à la déforestation, qui représentent environ 3,4 milliards de tonnes de $\mathrm{CO}_{2}$ par an en

\footnotetext{
1. www.globalcarbonproject.org/

2. $1 \mathrm{ppm}$ en volume est équivalent à $1 \mathrm{~cm}^{3}$ par $\mathrm{m}^{3}$ d'air.

3. www.esrl.noaa.gov/gmd/ccgg/trends/

4. www.cpc.ncep.noaa.gov/products/analysis_ monitoring/ensostuff/ensoyears.shtml
}

moyenne sur cette décennie. Même si cette source de $\mathrm{CO}_{2}$ reste encore mal quantifiée à ce jour, elle reste très inférieure à celle liée à l'utilisation des énergies fossiles.

Les puits naturels de $\mathrm{CO}_{2}$ que constituent l'océan et la végétation continentale ont, quant à eux, limité l'accumulation de $\mathrm{CO}_{2}$ dans l'atmosphère en absorbant respectivement 9,5 et 10,3 milliards de tonnes de $\mathrm{CO}_{2}$ par an en moyenne sur la période 2005-2014. À ce jour, seul le puits de $\mathrm{CO}_{2}$ océanique est relativement bien estimé. Les observations réalisées depuis les années 1990 et la modélisation ont permis de déterminer l'ampleur du puits océanique sur cette période. Par contre, le puits de $\mathrm{CO}_{2}$ dû à la végétation et aux sols est difficile à mesurer ; il est souvent déduit des autres termes du bilan global et reste donc très incertain.

En 2014, près de 36 milliards de tonnes de $\mathrm{CO}_{2}$ ont été émises par les activités

humaines. Cette année-là, la teneur de $\mathrm{CO}_{2}$ atmosphérique a franchi le seuil de 400 parties par million $\left(\mathrm{ppm}^{2}\right)$ dans l'hémisphère Nord, soit le niveau le plus élevé de ces 800000 dernières années. La hausse des émissions anthropiques de $\mathrm{CO}_{2}$ a pourtant été limitée à $0,6 \%$, une valeur nettement inférieure à la hausse annuelle moyenne sur la période 2003-2014, estimée à 2,4\%.

Ce fait est remarquable car, dans le passé, les hausses modestes des émissions anthropiques de $\mathrm{CO}_{2}$ ont toujours correspondu à des chocs économiques d'ampleur mondiale, ce qui n'est pas le cas en 2014.

L'année 2014 a aussi connu une forte absorption naturelle de $\mathrm{CO}_{2}$ par l'océan et la biosphère terrestre. Après avoir diminué pendant les années 1990 , l'absorption de carbone par l'océan Austral - une des principales régions d'absorption de $\mathrm{CO}_{2}$ de l'océan - a
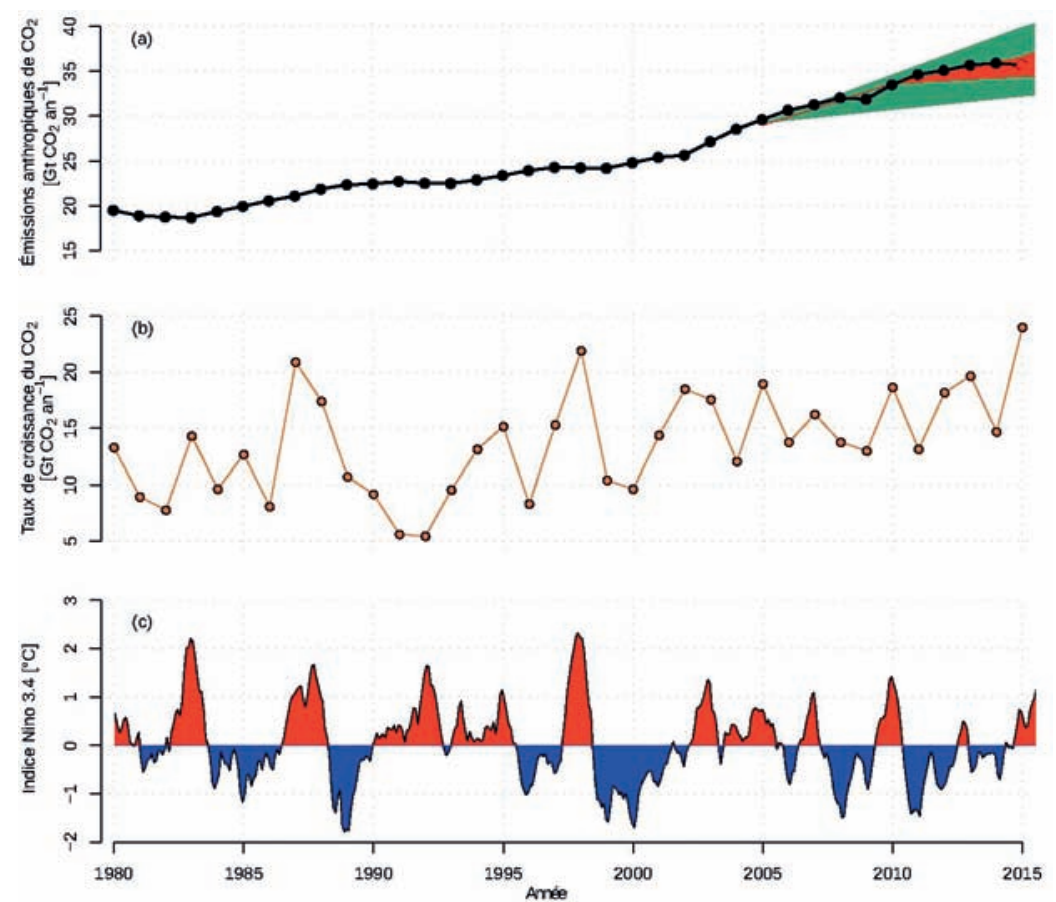

Figure 1. Évolution temporelle a) des émissions anthropiques de $\mathrm{CO}_{2}$ liées à l'utilisation des énergies fossiles et à la fabrication de ciment ; b) du taux de croissance de la teneur en $\mathrm{CO}_{2}$ de l'atmosphère ; c) de l'indice climatique El Niño 3.4. Les émissions anthropiques de $\mathrm{CO}_{2}$ sont issues de la compilation d'observations et de statistiques énergétiques du Global Carbon Project. La quantité de $\mathrm{CO}_{2}$ émise par les activités humaines en 2015 (point rouge) correspond à une estimation réalisée à partir des projections de croissance mondiale. Les projections d'émissions futures de $\mathrm{CO}_{2}$ anthropique effectuées lors des $4^{e}$ et $5^{\mathrm{e}}$ rapports du Giec sont représentées par les aplats de couleur verte et rouge. Le taux de croissance du $\mathrm{CO}_{2}$ atmosphérique est issu des observations de la Noaa ${ }^{3}$. L'indice climatique 4 El Niño 3.4 est calculé à partir d'observations de la température de la mer dans la région délimitée par $170^{\circ} \mathrm{W}-120^{\circ} \mathrm{W}$ et $5^{\circ} \mathrm{S}-5^{\circ} \mathrm{N}$. Les valeurs positives et négatives sont des écarts à la moyenne de cet indice et indiquent respectivement la présence des phénomènes El Niño et La Niña. 
retrouvé un niveau équivalent à celui des années 1990. Le renforcement des vents d'ouest au niveau du secteur pacifique de l'océan Austral a contribué à stimuler l'absorption de $\mathrm{CO}_{2}$ dans cet océan. L'absorption de $\mathrm{CO}_{2}$ par la végétation continentale est d'une amplitude équivalente à celle de 2011, la plus élevée depuis 60 ans. Cette absorption record semble être associée à des facteurs biogéochimiques tels que l'effet fertilisant du $\mathrm{CO}_{2}$ atmosphérique et des dépôts d'azote réactif, mais aussi des facteurs climatiques tels que le rallongement des saisons de croissance et l'absence de phénomène El Niño sur le Pacifique équatorial en 2014. Par leur action, l'océan et la végétation continentale ont permis de soustraire respectivement 10,7 et 15 milliards de tonnes de $\mathrm{CO}_{2}$ de l'atmosphère en 2014 , bien plus que la moyenne sur 2005-2014.

On estime que la quantité totale de $\mathrm{CO}_{2}$ pouvant encore être émise sans provoquer une hausse de la température mondiale supérieure à $2{ }^{\circ} \mathrm{C}$ se situe dans une fourchette de 500 à 1100 milliards de tonnes de $\mathrm{CO}_{2}$. Cela représente une échéance de l'ordre de 15 à 30 ans si les émissions anthropiques de $\mathrm{CO}_{2}$ continuent d'augmenter au rythme des dernières années.

Les premières estimations du Global Carbon Project pour l'année 2015 semblent indiquer une légère réduction des émissions anthropiques de $\mathrm{CO}_{2}$ d'environ $0,6 \%$ (figure 1a). La fourchette encadrant cette valeur, allant d'une réduction de $1,6 \%$ à une augmentation de $0,5 \%$, reflète la part d'incertitude associée aux projections de la croissance économique, mais également à des facteurs climatiques influant sur l'utilisation d'énergie comme les hivers plus doux aux moyennes latitudes. L'évaluation finale des émissions de $\mathrm{CO}_{2}$ à partir des statistiques énergétiques des pays sur l'année 2015 sera réalisée par le Global Carbon Project lors de la préparation du bilan de carbone sur la période 2006-2015.

Le suivi régulier de la teneur en $\mathrm{CO}_{2}$ de l'atmosphère indique que l'année 2015 est exceptionnelle à de nombreux égards, avec le plus fort taux de croissance annuel de $\mathrm{CO}_{2}$ atmosphérique (3,05 ppm, figure 1b) jamais enregistré depuis les premières observations en 1960 et le franchissement du seuil des 400 ppm à l'échelle du globe en 2015. Il est plus que probable que le retour du phénomène El Niño sur le Pacifique équatorial en 2015 (figure 1c) explique ce taux de croissance record, car des sursauts similaires mais d'une moindre ampleur ont pu être observés par le passé, notamment lors des phénomènes El Niño des années 1987 et 1998. Avec de telles variations annuelles pilotées par de nombreux facteurs naturels, l'impact d'une stabilisation, voire d'une diminution, des émissions anthropiques de $\mathrm{CO}_{2}$ sur la teneur en $\mathrm{CO}_{2}$ atmosphérique ne pourra être quantifié avec précision que dans quelques dizaines d'années.

Roland Séférian, Matthias Rocher

Centre national de recherches météorologiques, Météo-France / CNRS, Toulouse

Nicolas Metzl

Laboratoire d'océanographie et du climat : expérimentations et approches numériques, Institut

Pierre-Simon Laplace, Sorbonne Universités / CNRS / IRD / MNHN, Paris

Philippe Ciais

Laboratoire des sciences du climat et de l'environnement, Institut Pierre-Simon Laplace, CEA / CNRS / UVSQ, Gif-sur-Yvette

Dans le monde...

\section{5, une année " historique »}

Chaque année, l'Organisation météorologique mondiale (OMM) analyse l'évolution du climat, notamment de la température, grâce aux données de plusieurs organismes : le Centre Hadley du Met Office britannique et l'Unité de recherches sur le climat de l'université d'East Anglia, le Goddard Institute for Space Studies (Giss) de la Nasa et la National Oceanic and Atmospheric Administration (Noaa); elles sont corroborées par une analyse de la Japan Meteorological Agency.

Dans sa Déclaration sur l'état du climat mondial en 2015, l'OMM affirme que «l'année 2015 restera à de nombreux égards comme une année historique pour ce qui est du climat mondial. Année record en termes de chaleur, tant au niveau mondial que dans de nombreux pays, elle a vu tomber des records de température établis depuis le début des relevés modernes ».

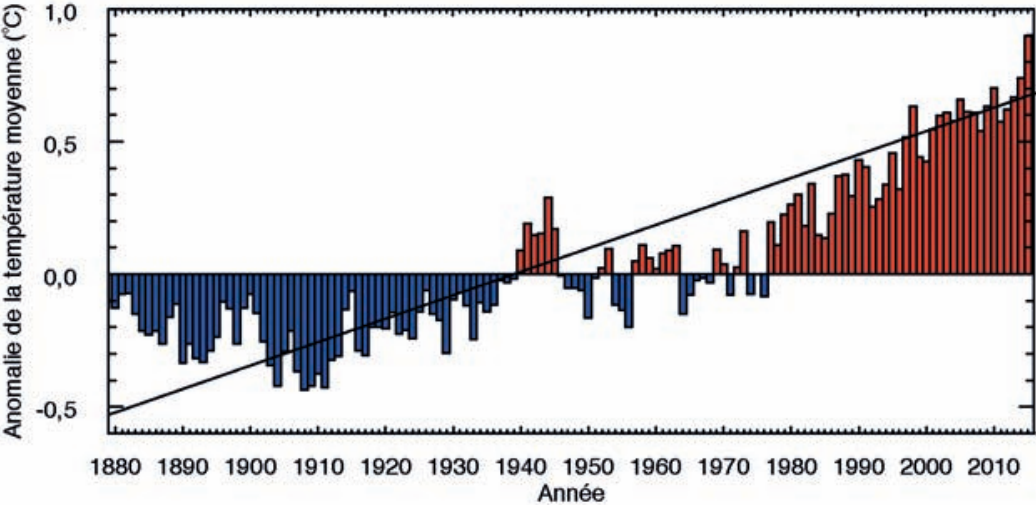

Figure 1. Anomalies de la température de surface mondiale 1880-2015. Source : Noaa.

Au niveau mondial, la température moyenne de 2015 est la plus élevée jamais enregistrée depuis qu'il y a une couverture suffisante de données pour calculer une moyenne à l'échelle du globe (figure 1). L'anomalie positive est de $0,76 \pm 0,09{ }^{\circ} \mathrm{C}$ par rapport à la normale 1961-1990 utilisée par l'OMM ; d'après la Noaa, elle est de $0,90{ }^{\circ} \mathrm{C}$ par rapport à la moyenne du $\mathrm{XX}^{\mathrm{e}}$ siècle $\left(13,9^{\circ} \mathrm{C}\right)$; le précédent record établi en 2014 est battu de $0,16{ }^{\circ} \mathrm{C}$. Tous les mois de 2015 ont connu une température excédentaire 